\title{
ISOMORPHISM BETWEEN CERTAIN SYSTEMS OF SIMPLE LINEAR GROUPS.
}

BY PROFESSOR L. E. DICKSON.

(Read before the American Mathematical Society, February 24, 1900.)

1. IN an article in the BuLletrs for July, 1899, giving the known finite simple groups, I made the conjecture that the simple quaternary hyperorthogonal group $H O\left(4, p^{2 n}\right)$ in the $G F\left[p^{2 n}\right]$ was isomorphic with the second hypoabelian group $S H\left(6,2^{n}\right)$, the orthogonal group $O\left(6, p^{n}\right)$, or the group $N S\left(6, p^{n}\right)$, according as $p^{n}$ is of the form $2^{n}, 4 l-1$, or $4 l+1$ respectively. For the case $* p^{n}=2$, and for the case $\dagger$ $p^{n}=3$, I have proven the conjecture true by setting up abstract groups holoedrically isomorphic with the linear groups in question. The calculations were necessarily long, so that the method of procedure would scarcely be adapted to the case of general $p^{n}$. From the correspondence of generators established in these two special cases, I have been led to the short proof for the general case given in this paper. The proof is based upon the theory of the second compound of a linear homogeneous group, as developed in the BuLLETIN for December 1898, and in the Transactions for January, 1900, pp. 91-96. In place of the hyperorthogonal group $H O\left(4, p^{2 n}\right)$, I employ the holoedrically isomorphic hyperabelian group $\$ H A\left(4, p^{2 n}\right)$, which contains as a subgroup the simple abelian group $A\left(4, p^{n}\right)$. The calculations appear to me to possess considerable elegance.

2. Theorem.-According as -1 is a square or a not-square in the $G F\left[p^{n}\right](p>2), H A\left(4, p^{2 n}\right)$ is holoedrically isomorphic with the group $\widehat{N S}\left(6, p^{n}\right)$ or $O\left(6, p^{n}\right)$.

Corresponding to every substitution of the subgroup $A\left(4, p^{n}\right)$ there is a senary linear homogeneous substitution of the second compound $H$ with the two invariants

$$
\psi \equiv Y_{12} Y_{34}-Y_{13} Y_{24}+Y_{14} Y_{23}, \quad Z \equiv Y_{12}+Y_{34} .
$$

To the hyperabelian substitution, in which $I$ belongs to the $G F\left(p^{2 n}\right)$,

$$
I \equiv\left(\begin{array}{llll}
I & 0 & 0 & 0 \\
0 & I^{-p^{n}} & 0 & 0 \\
0 & 0 & I^{-1} & 0 \\
0 & 0 & 0 & I^{p^{n}}
\end{array}\right]
$$

"Proc. of the Lond. Math. Soc., vol. 31, pp. 30-68.

†Transactions, vol. 1, No. 3 (July, 1900).

$\ddagger$ Proc. Lond. Math. Soc., vol. 31, pp. 30-68. 
there corresponds in the second compound $G$ of $H A\left(4, p^{2 n}\right)$, the substitution

$$
I^{\prime}:\left\{\begin{array}{lll}
Y_{12}^{\prime}=I^{-p^{n}+1} Y_{12}, & Y_{13}^{\prime}=Y_{18}, & Y_{14}^{\prime}=I^{p^{n}+1} Y_{14} \\
Y_{23}^{\prime}=I^{-p^{n}-1} Y_{28}, & Y_{24}^{\prime}=Y_{24}, & Y_{34}^{\prime}=I^{p^{n}-1} Y_{84}
\end{array}\right.
$$

Introducing in place of $Y_{12}$ and $Y_{34}$ the new indices $(p>2)$

$$
\xi_{1} \equiv \frac{1}{2}\left(Y_{12}-Y_{34}\right), \quad \xi_{6} \equiv \frac{-1}{2 J}\left(Y_{12}+Y_{34}\right),
$$

the reversion of which gives

$$
Y_{12} \equiv \xi_{1}-J \xi_{6}, \quad Y_{34} \equiv-\xi_{1}-J \xi_{6}
$$

the substitution $I^{\prime}$ takes the form

$$
I^{\prime \prime}:\left\{\begin{array}{c}
\xi_{1}^{\prime}=\frac{1}{2} \theta \xi_{1}+\frac{1}{2} J\left(I^{p^{n}-1}-I^{-p^{n}+1}\right) \xi_{6}, \\
\xi_{6}^{\prime}=\frac{1}{2 J}\left(I^{p^{n}-1}-I^{-p^{n}+1}\right) \xi_{1}+\frac{1}{2} \theta \xi_{6} \\
Y_{13}^{\prime}=Y_{13}, Y_{14}^{\prime}=I^{p^{n}+1} Y_{14}, \\
Y_{24}^{\prime}=Y_{24}, Y_{23}^{\prime}=I^{-p^{n}-1} Y_{23}, \\
\theta \equiv I^{p^{n}-1}+I^{-p^{n}+1} .
\end{array}\right.
$$

Since $\theta^{p^{n}}=\theta, \theta$ belongs to the $G F\left[p^{n}\right]$. The coefficient

$$
\frac{1}{2} J\left(I^{p^{n}-1}-I^{-p^{n}+1}\right) \text {, }
$$

whose $p^{n}$ th power is $\frac{1}{2} J^{p^{n}}\left(I^{1-p^{n}}-I^{-1+p^{n}}\right)$, belongs to the $G F\left[p^{n}\right]$ if and only if $J \equiv-J^{p^{n}}$, or $J^{p^{n}-1}=-1$. With this condition satisfied, the substitution $I^{\prime \prime}$ belongs to the $G F\left[p^{n}\right]$ and leaves invariant the new form of $\psi$, viz.,

$$
\psi^{\prime \prime} \equiv-\xi_{1}^{2}+J^{2} \xi_{6}^{2}-Y_{13} Y_{24}+Y_{14} Y_{23} .
$$

By the relation just assumed to hold,

$$
\left(J^{2}\right)^{p^{n}-1}=1, \quad\left(J^{2}\right)^{\left(p^{n}-1\right) / 2}=-1,
$$

so that $J^{2}$ belongs to the $G F\left[p^{n}\right]$, but is a not-square in it. It follows * that the group $G^{\prime \prime}$ given by the extension of $H^{\prime \prime}$

* "Determination of the structure of all linear homogeneous groups in a Galois field which are defined by a quadratic invariant," Amer. Jour. of Math., vol. 21, pp. 193-256. 
( $H$ when expressed in the indices $\xi_{1}, \xi_{6}, Y_{13}, Y_{14}, Y_{23}, Y_{24}$ ) by the substitution $I^{\prime \prime}$ is a subgroup of the group $Q$ of senary linear substitutions in the $G F\left[p^{n}\right]$ which leaves $\psi^{\prime \prime}$ invariant. The latter has a subgroup $Q^{\prime \prime}$ of index 2 which contains all the substitutions * $Q_{16}$. Further, $Q^{\prime \prime}$ is extended to $Q$ by any substitution $O_{16}$ which is not of the form $Q_{16^{\circ}}$. We proceed to prove that $G^{\prime \prime} \equiv Q^{\prime \prime}$. In order that $H^{\prime \prime}$ shall contain the substitution

$$
h: \quad Y_{14}^{\prime}=I^{p^{n}+1} Y_{14}, \quad Y_{23}^{\prime}=I^{-p^{n}-1} Y_{23},
$$

it is necessary and sufficient that $I^{p^{n}+1}$ be a square in the $G F\left[p^{n}\right]$, or

$$
I^{\left(p^{n}+1\right)\left(p^{n}-1\right) / 2}=+1
$$

$i$. e., that $I$ be a square in the $G F\left[p^{2 n}\right]$. If this condition be satisfied, $G^{\prime \prime}$ contains the product $K \equiv I^{\prime \prime} h^{-1}$, viz.,

$$
\begin{aligned}
& \xi_{1}^{\prime}=\frac{1}{2} \theta \xi_{1}+\frac{1}{2} J\left(I^{p^{n}-1}-I^{-p^{n}+1}\right) \xi_{6}, \\
& \xi_{6}^{\prime}=\frac{1}{2 J}\left(I^{p^{n}-1}-I^{-p^{n}+1}\right) \xi_{1}+\frac{1}{2} \theta \xi_{6},
\end{aligned}
$$

having determinant unity and leaving invariant $-\xi_{1}{ }^{2}+$ $J^{2} \xi_{6}^{2}$. Let

$$
\alpha \equiv \frac{1}{2}\left(I^{\frac{p^{n}-1}{2}}+I^{\frac{-p^{n}+1}{2}}\right), \quad \beta \equiv \frac{1}{2} J\left(I^{\frac{p^{n}-1}{2}}-I^{\frac{-p^{n}+1}{2}}\right) \text {. }
$$

We readily find that

$$
\begin{gathered}
\alpha^{2}-\frac{1}{J^{2}} \beta^{2}=1, \quad 2 \alpha \beta=\frac{J}{2}\left(I^{p^{n}-1}-I^{-p^{n}+1}\right), \\
2 \alpha^{2}-1=\frac{1}{2}\left(I^{p^{n}-1}+I^{-p^{n}+1}\right) .
\end{gathered}
$$

Hence $K=Q_{1,6}^{\alpha, \beta}, \alpha$ and $\beta$ being marks of the $G F\left[p^{n}\right]$. Inversely, if $\alpha$ and $\beta$ be any set of solutions in the $G F\left[p^{n}\right]$ of the first relation (6), we can determine a square $I$ in the $G F\left[p^{2 n}\right]$ which satisfies the last two relations (6). In fact, from them,

$$
I^{p^{n}-1}=2 \alpha^{2}-1+\frac{2 \alpha \beta}{J}=\left(\alpha+\frac{\beta}{J}\right)^{2}, I^{-p^{n}+1}=\left(\alpha-\frac{\beta}{J}\right)^{2} .
$$

The second follows from the first, since

$$
\left(\alpha+\frac{\beta}{J}\right)^{2}\left(\alpha-\frac{\beta}{J}\right)^{2}=\left(\alpha^{2}-\frac{\beta^{2}}{J^{2}}\right)^{2}=1 .
$$

\footnotetext{
* For the notations, see Amer. Jour. of Math., vol. 21, p. 207.
} 
To show that the first relation can be solved for a square $I$ in the $G F\left[p^{2 n}\right]$, we note that

$$
\left(\alpha+\frac{\beta}{J}\right)^{p^{n}+1}=\left(\alpha+\frac{\beta}{J^{p^{n}}}\right)\left(\alpha+\frac{\beta}{J}\right)=\alpha^{2}-\frac{\beta^{2}}{J^{2}}=1 .
$$

We have therefore shown that $G^{\prime \prime}$ contains every $Q_{1,6}^{\alpha, \beta}$. For $I$ a not-square in the $G F\left[p^{2 n}\right]$, the substitution $I^{\prime \prime}$ is the product of an $O_{1, \sigma}^{\rho, \sigma}\left(\right.$ not a $\left.Q_{16}\right)$ by $h$, neither of which separately belongs to $G^{\prime \prime}$. By introducing new indices $\xi_{2}, \xi_{3}$ in place of $Y_{14}, Y_{23}$, we may give $h$ the form $O_{2,3}^{\kappa, \lambda}$ (not a $\left.Q_{23}\right)$. It follows that $G^{\prime \prime} \equiv Q^{\prime \prime}$. In order to obtain the quotient group $H A\left(4, p^{2 n}\right)$, we consider as identical the products $S$ and $S T \equiv T S$, where $T$ is a hyperabelian substitution of determinant unity multiplying every index by $\tau$, so

that

$$
\tau^{4}=1, \quad \tau^{p^{n}+1}=1
$$

Hence, if $p^{n}=4 l+1$, the second compound $G^{\prime \prime}$ of $H A\left(4, p^{2 n}\right)$ is simple, and therefore holoedrically isomorphic with $N S\left(6, p^{n}\right)$. If $p^{n}=4 l-1, G^{\prime \prime}$ has a maximal invariant subgroup of order two, generated by the substitution changing the signs of all six indices, the quotient group being $O\left(6, p^{n}\right)$.

3. Theorem. - The simple groups $H A\left(4,2^{2 n}\right)$ and $S H\left(6,2^{n}\right)$ are holoedrically isomorphic.

Let $\lambda$ be a mark of the GF[2 $\left[2^{n}\right.$ such that the equation

$$
\sigma^{2}+\sigma+\lambda^{2}=0
$$

is irreducible in the $G F\left[2^{n}\right]$. The condition on $\lambda$ for the irreducibility of (6) is readily given (American Journal of Mathematics, 1. c., p. 224) and can always be satisfied. Its roots are then $\sigma$ and $\sigma^{2^{n}}$ where

$$
\sigma+\sigma^{2^{n}}=-1
$$

We apply to the substitution $I^{\prime}$ of $\$ 2$ the transformation of indices

$$
Y_{12} \equiv \lambda^{\frac{1}{2}} \xi_{1}-\sigma \lambda^{-\frac{1}{2} \xi_{6}}, \quad Y_{34} \equiv \lambda^{\frac{1}{2} \xi_{1}}-\lambda^{\frac{3}{2}} \sigma^{-1} \xi_{6},
$$

which gives reciprocally, for $p=2$,

$$
\xi_{1} \equiv \lambda^{\frac{3}{2}} \sigma^{-1} Y_{12}+\lambda^{-\frac{1}{2}} \sigma \dot{Y}_{34}, \quad \xi_{6}=\lambda^{\frac{1}{2}}\left(Y_{12}+Y_{34}\right)
$$

Multiplying $I^{\prime}$ by $h^{-1}$ ( $h$ defined as in $\$ 2$ ), we obtain the substitution :

$$
T^{\prime}: \quad Y_{12}^{\prime}=\tau Y_{12}, \quad Y_{34}^{\prime}=\tau^{-1} Y_{34} \quad\left(\tau=I^{-2^{n}+1}\right) .
$$


Expressed in terms of the new indices $\xi_{1}, \xi_{6}$ defined by (9), $T^{\prime}$ becomes

$$
T^{\prime \prime}:\left\{\begin{array}{l}
\xi_{1}^{\prime}=\left(\lambda^{2} \sigma^{-1} \tau+\sigma \tau^{-1}\right) \xi_{1}-\lambda\left(\tau+\tau^{-1}\right) \xi_{6}, \\
\xi_{6}^{\prime}=\lambda\left(\tau+\tau^{-1}\right) \xi_{1}-\left(\lambda^{2} \sigma^{-1} \tau^{-1}+\sigma \tau\right) \xi_{6} .
\end{array}\right.
$$

We readily prove that the coefficients of $T^{\prime \prime}$ belong to the $G F\left[2^{n}\right]$. Since $\tau \equiv I^{-2^{n}+1}$, we find that $\tau+\tau^{-1}$ equals its $2^{n}$ th power and is therefore in the $G F\left[2^{n}\right]$. Furthermore, by (6),

$$
\lambda^{2} \sigma^{-1} \tau+\sigma \tau^{-1} \equiv \tau+\sigma\left(\tau+\tau^{-1}\right) \quad(\bmod 2) .
$$

The latter equals its $2^{n}$ th power since, by (7), we have

$$
\begin{aligned}
& \tau^{2^{n}}-\tau+\left(\sigma^{2^{n}}-\sigma\right)\left(\tau+\tau^{-1}\right)=\left(\tau+\tau^{-1}\right)\left(\sigma^{2^{n}}-\sigma+1\right) \\
& {[\equiv 0 \quad(\bmod 2) \text {. }}
\end{aligned}
$$

Changing $\tau$ into $\tau^{-1}$, it follows that $\lambda^{2} \sigma^{-1} \tau^{-1}+\sigma \tau$ belongs to the GF[2 $\left.2^{n}\right]$.

By the transformation (8), $\psi$ becomes, on applying (6),

$$
\psi_{1} \equiv \lambda\left(\xi_{1}^{2}+\xi_{6}^{2}\right)+\xi_{1} \xi_{0}-Y_{18} Y_{24}+Y_{14} Y_{23} .
$$

Hence the group $\Gamma^{\prime \prime}$ given by the extension of the group $H^{\prime \prime}$ by $T^{\prime \prime}$ is a subgroup of $S H\left(6,2^{n}\right)$. Evidently $H^{\prime \prime}$ contains all the substitutions of $S H\left(6,2^{n}\right.$ ) which leave $\xi_{6}$ (as well as $\left.\psi_{1}\right)$ invariant. But $S H\left(6,2^{n}\right)$ is given by the extension of $H^{\prime \prime}$ by the substitutions $*$

$$
O_{1,6 !}^{\alpha, \beta^{\prime}}:\left\{\begin{array}{l}
\xi_{1}^{\prime}=\alpha \xi_{1}+\lambda(\alpha+\beta) \xi_{6} \\
\xi_{6}^{\prime}=\lambda(\alpha+\beta) \xi_{1}+\beta \xi_{6}
\end{array} \quad\left[\alpha \beta+\lambda^{2}\left(\alpha^{2}+\beta^{2}\right)=1\right] .\right.
$$

The totality of substitutions $O_{1,6}^{\alpha, \beta}$ is identical with the totality of substitutions $T^{\prime \prime}$ when $\tau$ takes all possible values for which $\tau^{2^{n}+1}=1$. In fact, if we set

$$
\alpha=\lambda^{2} \sigma^{-1} \tau+\sigma \tau^{-1}, \quad \beta=\lambda^{2} \sigma^{-1} \tau^{-1}+\sigma \tau,
$$

we get by (6),

$$
\alpha+\beta=\tau+\tau^{-1}, \quad \alpha \beta+\lambda^{2}\left(\alpha^{2}+\beta^{2}\right)=1 .
$$

Hence every $T^{\prime \prime}$ is of the form $O_{1,6}^{\alpha, \beta}$. Inversely, if we set

$$
\tau=\alpha+\sigma(\alpha+\beta), \text { whence } \tau^{-1}=\beta+\sigma(\alpha+\beta),
$$

we see that every $O_{1,6}^{\alpha, \beta}$ is of the form $T^{\prime \prime}$.

* Amer. Jour. of Math., vol. 21, pp. 225-233. For uniformity of notation, I here use $O_{1,6}^{\alpha, \beta}$ in place of $O_{1}^{\alpha, \beta}$ of p. 226, replacing $\eta_{1}$ by $\xi_{\theta}$. 
It follows that $\Gamma^{\prime \prime}$ is identical with $S H\left(6,2^{n}\right)$. But $\Gamma^{\prime \prime}$ is holoedrically isomorphic with $G_{p=2}$ and therefore with $H A\left(4,2^{2 n}\right)$, whose second compound is $G_{p=2}$.

4. NotE.- It appears that the quaternary transformation group which naturally corresponds to the finite group $H O\left(4, p^{2 n}\right)$ is not continuous.

The University of Texas, January 27, 1900.

\section{THE HESSIAN OF THE CUBIC SURFACE. II.}

BY DR. J. I. HUTCHINSON.

(Read before the American Mathematical Society, February 24, 1900.)

THe aim of the following paper is to extend the results of a previous article on the same subject (Bulletin, March, 1899, p. 282) by determining all the quintic and sextic curves on the Hessian of the cubic surface, and giving some theorems connected with them, and with the quartic curves already determined.

I will write the equation of the Hessian in the form

$$
F \equiv x y z u+w y z u+w x z u+w x y u+w x y z=0,
$$

where $w, x, y, z, u$ are connected by the relation

$$
a w+b x+c y+d z+e u=0,
$$

in which $a, b, c, d, e$ are arbitrary constants.

As already shown, the surface $F$ contains three classes of biquadratic curves, viz.:

$a_{1}$. A class containing 15 families which lie on 30 families of cones, all the cones of the same family cutting $F$ in two lines and tangent along a third.

$\alpha_{2}$. A class containing 30 families of curves lying on 30 families of cones tangent to $F$ along two lines.

$\alpha_{3}$. A class containing 15 families of curves determined by as many families of quadrics each intersecting $F$ in a gauche quadrilateral, and by 30 families of quadrics each meeting $F$ in two lines and a conic.

Consider the family of $\alpha_{1}$ determined by the cones

$$
\begin{aligned}
& A_{1} \equiv x(w+y)+\lambda w y=0, \\
& A_{1}^{\prime} \equiv x(z+u)+(1-\lambda) z u=0 .
\end{aligned}
$$

